\title{
QuickStats
}

\section{Percentage Distribution of Gestational Age in Weeks for Infants Who Survived to Age 1 Year and Infants Who Died Before Age 1 Year - National Vital Statistics System, United States, 2014}

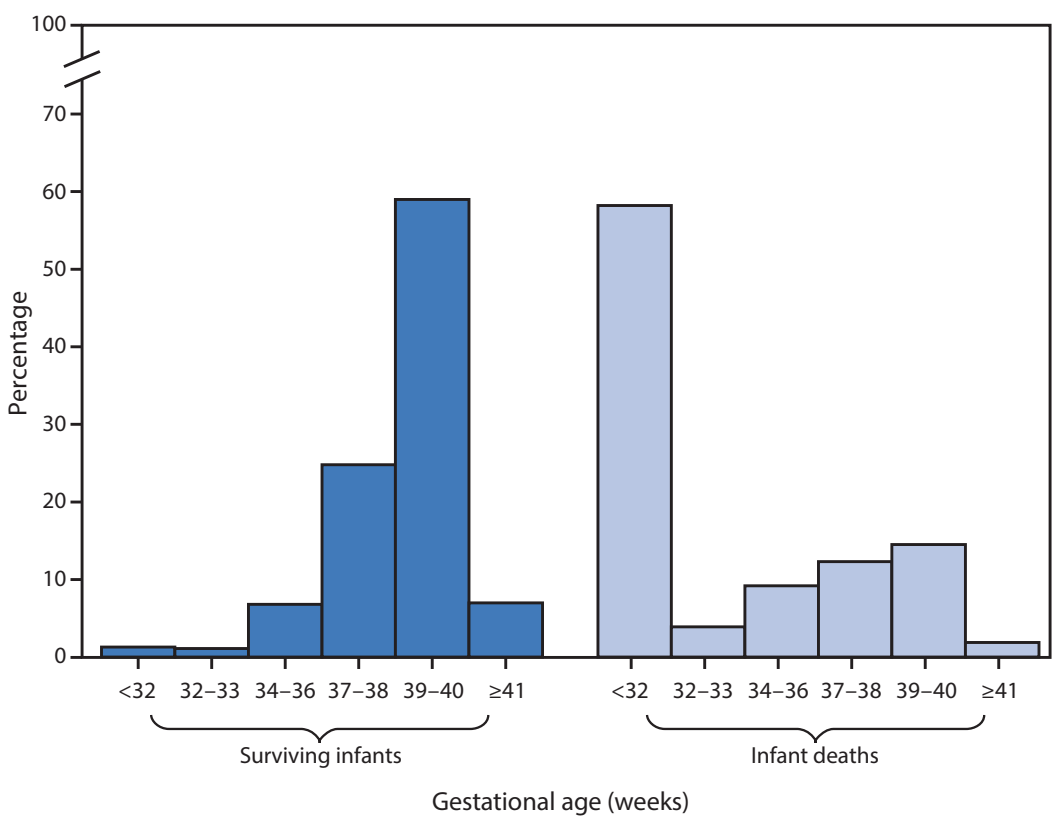

Infants who do not survive the first year of life are more likely to be born at earlier gestational ages. In $2014,66 \%$ of infants who survived to age 1 year were delivered at full term or later ( $\geq 39$ completed weeks) compared with $16 \%$ of infants who died before reaching age 1 year. Fifty-eight percent of infants who died before age 1 year were delivered at $<32$ weeks gestation compared with only $1 \%$ of infants who survived to age 1 year.

Source: National Vital Statistics System, Linked Birth and Infant Death Data. https://www.cdc.gov/nchs/nvss/linked-birth.htm. Reported by: Anne K. Driscoll, PhD, adriscoll1@cdc.gov, 301-458-4341. 